\title{
Accelerating Evolution via Egalitarian Social Learning
}

\author{
Wesley Tansey, Eliana Feasley, Risto Miikkulainen \\ Dept. of Computer Science, The University of Texas at Austin \\ 1 University Station C0500, Austin, TX, USA \\ \{tansey, elie, risto\}@cs.utexas.edu
}

\begin{abstract}
Social learning is an extension to evolutionary algorithms that enables agents to learn from observations of others in the population. Historically, social learning algorithms have employed a student-teacher model where the behavior of one or more high-fitness agents is used to train a subset of the remaining agents in the population. This paper presents ESL, an egalitarian model of social learning in which agents are not labeled as teachers or students, instead allowing any individual receiving a sufficiently high reward to teach other agents to mimic its recent behavior. We validate our approach through a series of experiments in a robot foraging domain, including comparisons of egalitarian social learning with baseline neuroevolution and a variant of studentteacher social learning. In a complex foraging task, ESL converges to near-optimal strategies faster than either benchmark approach, outperforming both by more than an order of magnitude. The results indicate that egalitarian social learning is a promising new paradigm for social learning in intelligent agents.
\end{abstract}

\section{Categories and Subject Descriptors}

I.2.6 [Computing Methodologies]: Artificial IntelligenceLearning, Problem Solving, Control Methods, and Search

\section{Keywords}

Social Learning, Evolutionary Algorithms, Artificial Life, Cultural Algorithms, On-line Evolution

\section{INTRODUCTION}

Evolutionary algorithms (EAs) [11] evaluate agents either in isolation or in direct competition with a subset of the other members of the population. Social and cultural learning algorithms [21] extend EAs by enabling agents to leverage observations of other members of the population to improve their own performance during their lifetime. By learning from others without having to directly experience

Permission to make digital or hard copies of all or part of this work for personal or classroom use is granted without fee provided that copies are not made or distributed for profit or commercial advantage and that copies bear this notice and the full citation on the first page. To copy otherwise, to republish, to post on servers or to redistribute to lists, requires prior specific permission and/or a fee.

GECCO'12, July 7-11, 2012, Philadelphia, Pennsylvania, USA.

Copyright 2012 ACM 978-1-4503-1177-9/12/07 ...\$10.00. or acquire knowledge, social learning algorithms have been able to improve the learning rate of EAs in many challenging domains $[9,21,12,19,17,26,2,8]$.

Traditionally in social learning algorithms, each agent is either a student or a teacher [20,3]. All actions of the teacher agents are considered to be good examples from which to learn, as they are derived from a high-fitness strategy (i.e., the teacher's policy). However, an agent with high overall fitness may not always choose good actions and agents with low overall fitness may actually perform well in some limited scenarios. Filtering potential observations based on their own merit may therefore be more appropriate and lead to both improved learning rate and stronger final strategies.

This paper presents Egalitarian Social Learning (ESL) as an alternative to the student-teacher paradigm. Agents in ESL are divided into subcultures at the start of each generation and can learn from any other agent in their subcultural group. Learning examples are determined by a user-defined acceptability function that filters out examples leading to low rewards. When an action is accepted, agents mimic it in order to learn a policy similar to that of the observed agent. ESL differs from other social learning algorithms in that the quality of a training example is measured by the reward received rather than the fitness of the agent generating the example.

We validate our algorithm in a well-known foraging domain $[9,12,26,2,8]$ in which agents must learn to navigate the world efficiently, gathering nutritious food while avoiding poisonous food. The empirical results indicate that egalitarian social learning is able to quickly find near-optimal strategies more than an order of magnitude faster than either a student-teacher variant or baseline neuroevolution in a complex domain. Additionally, the use of subcultures in ESL is shown to promote behavioral diversity and prevent premature convergence to sub-optimal strategies that would otherwise emerge if agents observed all individuals in the population.

This paper makes the following novel contributions:

- An egalitarian social learning algorithm, in which all individuals in a subculture observe, learn from, and teach each other:

- A behavioral diversity analysis of two egalitarian social learning variants;

- A performance comparison between ESL and traditional student-teacher social learning.

The remainder of the paper is structured as follows. Background information and prior work is discussed in Section 
2. Section 3 details the how the Egalitarian Social Learning algorithm works. Section 4 describes both the experimental setup and the foraging domain in detail. Section 5 presents experimental results. A discussion of the results and planned future work are described in Section 6. Section 7 presents conclusions.

\section{BACKGROUND}

In this section, background information and motivation for social learning is provided, as well as an introduction to the evolutionary framework underlying ESL.

\subsection{Social Learning}

One explanation for the evolution of large brains in primates is the social intelligence hypothesis [6, 15], which states that the need to handle complex social behaviors was the primary selection pressure driving the increase in brain size. The cultural intelligence hypothesis extends this concept solely to humans, stating that our brains evolved to handle the specific challenge of culture creation and social learning [25]. These hypotheses are currently the most widely accepted explanations for the evolution of the human brain among evolutionary biologists and cognitive scientists [14], and have been supported by strong empirical evidence in recent years [13].

Cultural and social learning algorithms [21] model this biological mechanism in multi-agent systems by designating teacher agents that propagate knowledge and train other agents in the population. These techniques effectively enhance EAs with a hierarchical structure (i.e., students and teachers) that makes it possible to discover suitable actions to use as training examples and target individuals to train. Typical cultural algorithms therefore capture the ability of humans to learn from formal instruction by an expert, but they do not fully model all forms of learning from observation in primates. For instance, human brains contain mirror neurons [1] that activate when other humans are observed interacting with the world, in effect mirroring the observed human's action internally. Such processes enable humans to learn socially without concern for the status or expertise of the observed individual.

\subsection{Egalitarianism}

Egalitarianism refers to any philosophy that advocates treating all individuals in a population as equals, regardless of such factors as background and status [4]. In huntergatherer societies, egalitarianism is a common paradigm for managing daily activities and organizing social structures [5]. It is likely that this lack of hierarchy and strict maintenance of equality has been pivotal in the development of human society and in separating humans from other primates [10].

\subsection{Related Work}

Enhancing EAs with social learning is a flourishing area of research with a long and successful track record. This section highlights relevant prior work and explains how ESL differs from previous efforts.

Cultural algorithms [21] have been used frequently in Particle Swarm Optimization (PSO) [16]. These algorithms maintain a "belief space" representing different categories of knowledge that the population has learned. New individuals are trained using this belief space in a student-teacher paradigm. In contrast, ESL agents maintain no separate repository of formal knowledge, instead learning from observations of others during their lifetime.

Interestingly, ESL could be seen as a memetic algorithm (MA) [18]. As originally defined by Dawkins [7], a meme is a "unit of imitation in cultural transmission" including "tunes, ideas, catch-phrases, clothes fashions, ways of making pots or of building arches". Knowledge propagation in ESL particularly relates to the latter - ways of making pots or building arches are similar to strategies for gathering food in that they all are instances of transmitting specific strategies for performing skilled tasks. Thus, each acceptable learning example could be considered a meme being shared within the agent's subculture. Nevertheless, traditional MAs [19], including similar Lamarckian evolution methods for evolving recurrent neural networks [17], focus on using local improvement heuristics and typically rely on off-line transmission of memes via mating and genetic recombination. However, agents in ESL transmit memes on-line as they are generated and observed during the evaluation process. Thus, while we could classify ESL as an MA, we believe the distinct transmission and learning techniques employed by the algorithm better characterize it as a social learning method.

The ability of social learning to improve agents in a foraging domain has been explored by several researchers in recent years. Denaro et. al. [9] used a student-teacher model of cultural evolution without genetic inheritance and demonstrated that the population will continue to improve if Gaussian noise is added to the training examples. The NEW TIES system $[12,26]$ simulates a steady-state evolution of decision tree agents where at each step the teacher agents probabilistically transmit their decisions and students probabilistically incorporate this knowledge. Acerbi et. al. [2] use social learning to train embodied agents to mimic the behaviors of more experienced agents. Finally, de Oca et. al. [8] propose a methodology for incremental social learning in PSO to update Q-learning [27] value functions by randomly selecting two individuals from the population and combining their values for a given update. While all of these studies are closely related and motivated by similar biological processes as ESL, they fundamentally all rely on the concept of students and teachers, and perform either no filtering or a reward-agnostic filtering of state-action pairs to be used in updating the population.

\subsection{Evolutionary Framework}

NeuroEvolution of Augmenting Topologies (NEAT)[24] is an evolutionary algorithm that generates recurrent neural networks. Through a process of adding and removing nodes and changing weights, NEAT evolves genomes that decode into networks. In every generation, those networks with the highest fitness reproduce, while those with the lowest fitness are unlikely to do so. NEAT maintains genetic diversity through speciation and encourages innovation through explicit fitness sharing.

In the foraging domain, NEAT is used to generate a population of individual neural networks that control agents in the world. The input to each network is the agent's sensors, and the outputs control the agent's velocity and orientation. The fitness of each network is determined by the success of the agent it controls - over the course of a generation, networks that control agents who eat a good deal of rewarding food and very little poison will have high fitness and those 


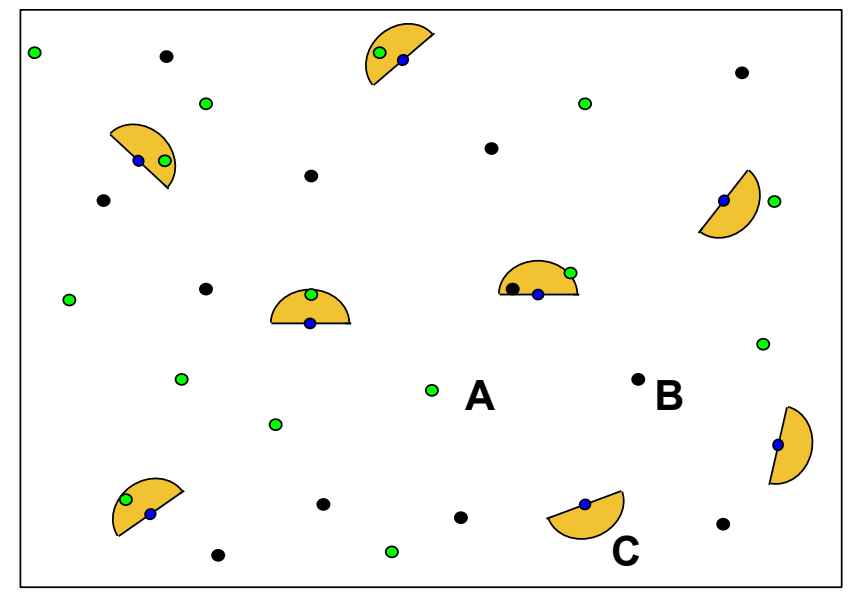

Figure 1: The foraging domain. This domain is a foraging world in which agents gain fitness by consuming plants that they approach. A) A piece of nutritious food which increases fitness. B) A piece of poisonous food which decreases fitness. C) An agent, the architecture of which is described in Figure 2 .

that control agents with less wise dietary habits will have low fitness.

In standard NEAT, the networks that are created do not change within one generation. To facilitate social learning, we must perform backpropagation [22] on the networks that NEAT creates in order to train agents on accepted examples. Since NEAT networks are recurrent, ESL enhances NEAT with backpropagation capabilities using the backpropagation through time algorithm [28].

The final fitness of each phenome, then, reflects the performance of the individual that used that phenome and elaborated on it over the course of a generation. This elaboration drives evolution in two alternate ways. In Darwinian evolution, the changes that were made to the phenome only affect selection and are not saved; in Lamarckian, the genome itself is modified.

\section{EGALITARIAN SOCIAL LEARNING}

Algorithm 1 presents the on-line, generational learning algorithm for Egalitarian Social Learning. ESL is designed for domains where an agent receives multiple rewards throughout its lifetime. Agents maintain a sliding window of their most recent states, actions, and rewards that serve as training examples for observing agents. When an agent receives a reward, other agents observe the event and determine if it qualifies as an acceptable training example. The key insight in ESL is that the agents should focus on the event itself to determine if the observed actions should be emulated. The overall fitness of the acting agent is not considered, as it is possible that a low-fitness strategy may perform high-quality actions in certain circumstances.

To promote diversity and prevent the population from prematurely converging to local optima, all agents are divided into observational groups called subcultures. At each timestep, each agent observes its state, responds with a desired action, and receives a resulting reward. The memory of an individual agent is thus a tuple containing its most recent

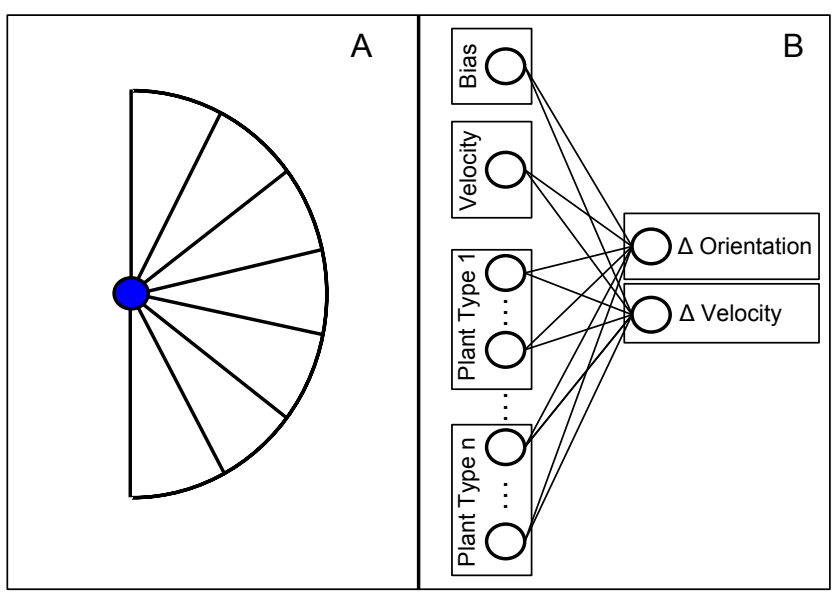

Figure 2: The architecture of our foraging agents. A) Each agent has a $180^{\circ}$ field of vision, represented as eight sensors for each plant type. B) Each agent is controlled by a neural network taking the agent's current velocity and sensor activations as inputs and outputting the desired change in orientation and velocity.

state, action, and reward. Each agent's memory is evaluated by all other members of its subculture using a user-defined acceptability function. The role of this function is to filter out low-quality examples and focus the agents on learning only from actions that will lead to strong strategies. When an agent's recent memory is judged as acceptable, it is then used as a teaching example for all other agents in its subculture.

The next section presents the experimental domain used to evaluate the efficacy of Egalitarian Social Learning.

\section{EXPERIMENTAL SETUP}

This section describes the evaluation domain, including the inputs and outputs that agents receive, and the common parameters across all experiments. ${ }^{1}$

\subsection{The Foraging Domain}

The domain used to evaluate ESL is a foraging world in which agents move freely on a continuous toroidal surface. The world is populated with various plants, some of which are nutritious and bear positive reward, while others are poisonous and bear negative reward. These plants are randomly distributed over the surface of the world. The foraging domain is non-competitive and non-cooperative; each agent acts independently of all other agents, with the exception of the teaching signals that pass between them. At the start of each generation, all individuals begin at the center of the world, oriented in the same direction, and confronted with the same plant layout and configuration. Every agent then has a fixed number of time steps to move about the surface of the world eating plants - which happens automatically when an agent draws sufficiently close to one- before the evaluation is over. Figure 1 shows an example of a foraging world with two types of plants and eight agents.

\footnotetext{
${ }^{1}$ All code for these experiments is available online at http://github.com/tansey/social-learning.
} 


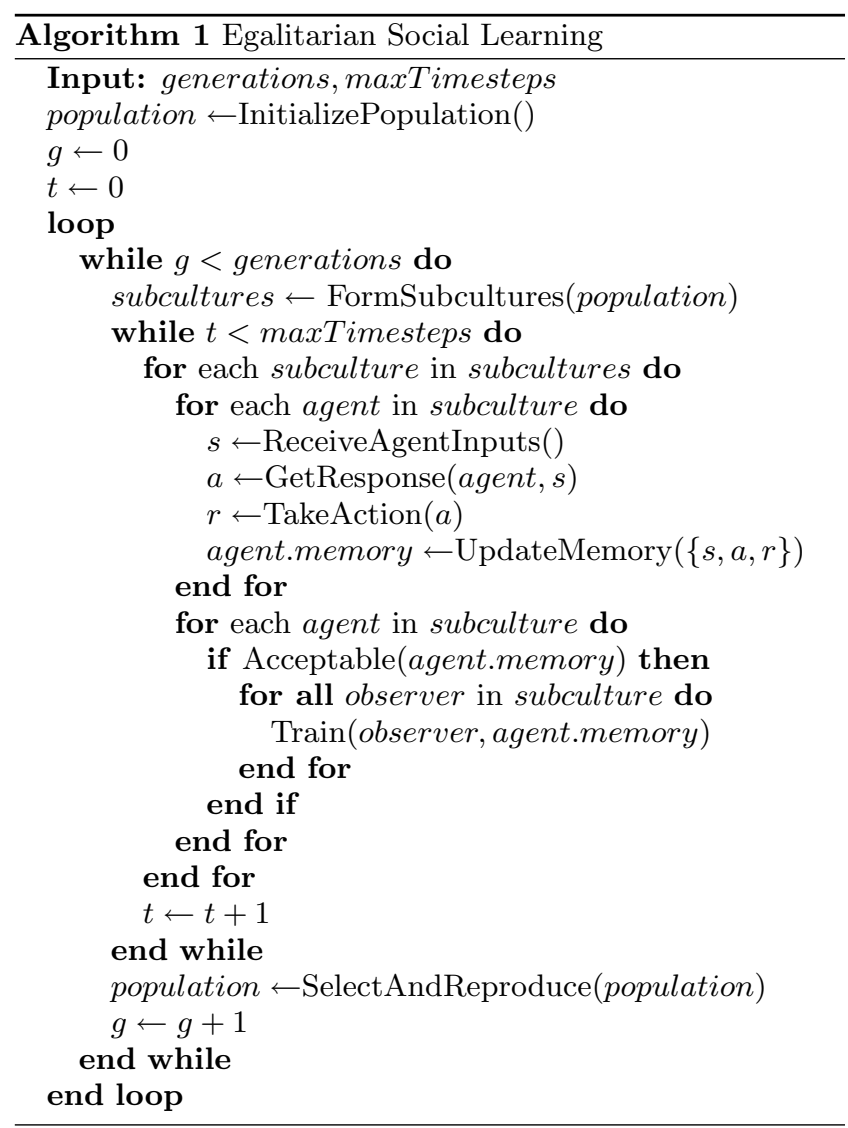

\subsection{Sensors and Outputs}

Agents "see" plants within a $180^{\circ}$ field of vision with a 100-unit horizon via a collection of sensors. Each agent has eight sensors for each type of plant, with each sensor covering a different $22.5^{\circ}$ sector of the $180^{\circ}$ ahead of the agent. Agents cannot see other individuals or plants they have already eaten - all they can see is edible food. The strength of the signal generated by each plant is proportional to its proximity to the agent. Agents also have a sensor by which they can detect their current velocity. As agents can only turn up to $30^{\circ}$ in a given timestep, knowledge of velocity is necessary for agents to accurately plan optimal trajectories (e.g., agents may need to slow down in order to avoid overshooting a plant). Each agent is controlled by an artificial neural network that maps from the agent's sensor readings to the desired change in orientation and velocity. Figure 2 shows the architecture of a foraging agent.

\subsection{Common Parameters}

Two separate configurations of the robot foraging world are used in the experiments. The experiments presented in Figures 3, 4, 5, and 8 use a "simple" world where the toroidal surface is 2000 by 2000 units, with a single plant type of value 100 and 100 randomly distributed instances of the plant. In this world, the agents have a straightforward task of learning to navigate efficiently and gather as many plants as possible.

The remaining set of experiments uses a second, more complex world to evaluate performance. The "complex" world has a surface of 500 by 500 units, with five different plant types of value $-100,-50,0,50$, and 100 . For

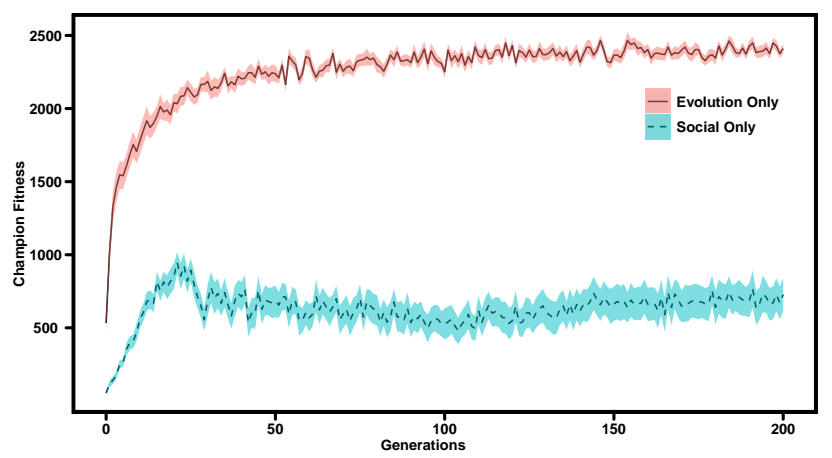

Figure 3: A comparison of the two learning components of ESL in isolation. The evolution-free social learning algorithm collapses after several generations, while neuroevolution alone slowly converges to a stable strategy. Egalitarian social learning combines both methods.

each plant type, 20 instances are created and randomly distributed across the surface. This world presents the agents with a more difficult task as they must efficiently gather nutritious food while simultaneously avoiding the poisonous food.

In all four experiments, 100 different agents are created in each generation. All networks are initialized with fullyconnected weights with no hidden neurons and a learning rate of 0.1 is used when performing backpropagation. Agents automatically eat any plant within five units. Each evaluation lasts 1000 timesteps and the results for each experiment are the average of 30 independent runs. In all plots, error bands denote standard error. The acceptability function for all experiments is to learn from any action yielding a positive reward.

The next section describes each experiment and its outcome in detail.

\section{RESULTS}

Four main experiments were conducted: 1) determining the baseline performance of egalitarian social learning and simple neuroevolution, each in isolation, 2) measuring the performance of a monocultural ESL in Darwinian and Lamarckian evolutionary paradigms, 3) evaluating whether subcultural learning improves performance compared to having a monoculture population, and 4) comparing ESL to a student-teacher learning algorithm in both simple and complex foraging tasks. The first three experiments examine the efficacy of the various components of the ESL algorithm while the fourth experiment serves to validate the final algorithm against a variant of a related approach in the literature. The experimental results show that ESL is able to evolve high-quality strategies with substantially fewer agent evaluations in the complex task than previous algorithms.

\subsection{Egalitarian Learning vs. Neuroevolution}

The first experiment establishes a baseline for understanding the ability of our two component methods, social learning and neuroevolution, to evolve high-quality strategies in isolation. To evaluate social learning in isolation, a monocultural population of 100 randomly initialized neural networks 


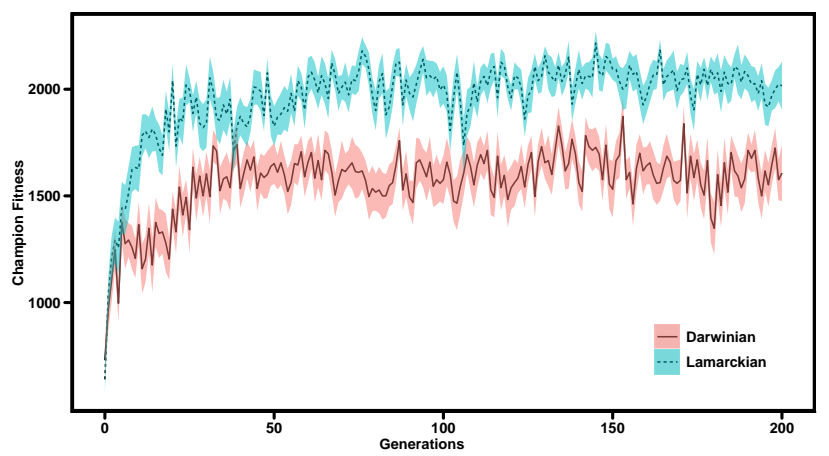

Figure 4: The effects of Darwinian and Lamarckian evolution when using a monocultural variant of ESL. While both evolutionary paradigms converge rapidly Lamarckian evolution is more effective than Darwinian in the foraging domain. Consequently, Lamarckian evolution is the paradigm used in all remaining experiments.

is created and evaluated without selection or reproduction for $200 \mathrm{~K}$ time steps. After every 1000 time steps, the population champion is recorded and the population fitness and world are reset. This is treated as a "generation" in order to compare it to baseline neuroevolution.

Figure 3 shows the results of the experiment. While evolutionfree social learning alone is able to improve initially, after several epochs a regression-to-the-mean effect is observed in which the entire population converges to a mediocre average score. A similar effect has been observed in previous social learning experiments [9], suggesting that some form of mutation is needed to prevent population collapse. In contrast, neuroevolution evolves solutions with fitnesses two to three times higher than that of egalitarian social learning alone after approximately 50 generations.

Having validated that social learning alone is not sufficient, the next experiment compares the performance of social learning when used as an enhancement to two different forms of simulated evolution.

\subsection{Darwinian vs. Lamarckian Evolution}

Genetic inheritance paradigms in evolution fall into one of two main categories: Darwinian and Lamarckian. In Darwinian evolution, individual genomes are fixed and any knowledge or abilities gained during their lifetimes are not passed on to their offspring at birth. By contrast, in Lamarckian evolution an individual's genome changes as it learns throughout its life, and these changes are passed on to each of its offspring. In the context of neuroevolution, this corresponds to whether the changes in each individual's neural network weights, as a result of social learning, are propagated to their genome at the end of the generation.

Figure 4 shows the results of applying a monocultural egalitarian social learning algorithm to the foraging domain in both the Lamarckian and Darwinian paradigms. The performance of both algorithms quickly converges, with Lamarckian reaching a higher-fitness solution than Darwinian evolution. In the context of on-line evolutionary learning algorithms, previous work [29] showed that Darwinian evolution is likely to be preferable to Lamarckian evolution in dynamic

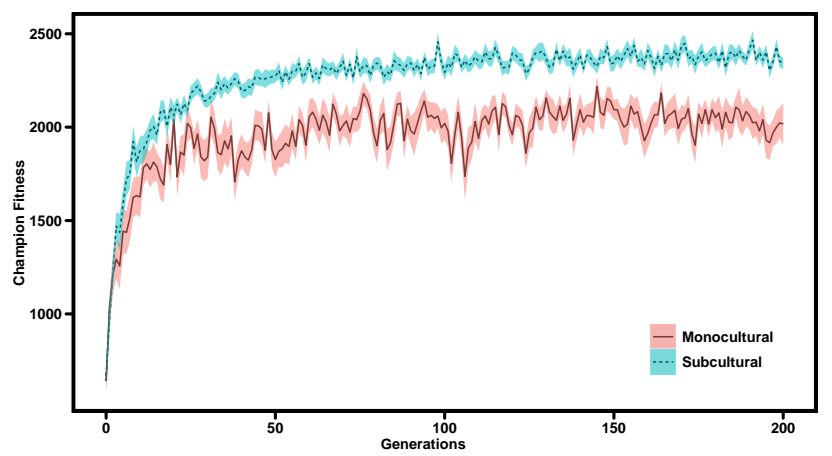

Figure 5: Monocultural agents learning from the entire population and subcultural agents learning only from their subcultures. Subcultural agents outperform monocultural agents, converging to a much higher ultimate fitness.

environments where adaptation is essential and the Baldwin effect [23] may be advantageous. However, as adaptation is not necessary for foraging agents (i.e., the rewards of each plant type are the same in every generation), in this experiment Lamarckian evolution outperforms Darwinian evolution.

Nevertheless, in both cases performance converges to a lower score than that of simple neuroevolution (Figure 3). The next experiment presents the results of introducing subcultures into the egalitarian model to overcome the tendency to converge.

\subsection{Monocultural vs. Subcultural Learning}

On one hand, monocultural Lamarckian social learning is able to find good results quickly, while on the other hand, it is likely to provide redundant information that may result in getting stuck in local optima.

In order to address this problem, we introduce a subcultural version of egalitarian social learning designed to promote and protect diversity. At the start of each generation, the population is divided into 10 subcultures of 10 agents each, with each agent's subculture decided at random. During the evaluation, agents only teach and learn from other agents in their own subculture. In addition to increasing behavioral diversity relative to learning from the entire population, this variant also has the appealing practical advantage that it decreases the worst-case number of iterations of backprop. For instance, in a population of 100 individuals with ten subcultures, an accepted training example is propagated to only 9 other agents, compared to 99 agents for the same example in a monocultural algorithm.

Figure 5 shows results comparing monocultural and subcultural learning. Subcultural learning has a steeper learning curve than the monocultural method. When every mutated organism has the opportunity to train every other, as is the case in monocultural learning, the entire population may be negatively impacted by any one individual. By preventing agents that lead the population towards local optima from impacting the remainder of the population, subcultural learning provides safety and protection from premature convergence. Because subcultural evolution is more 


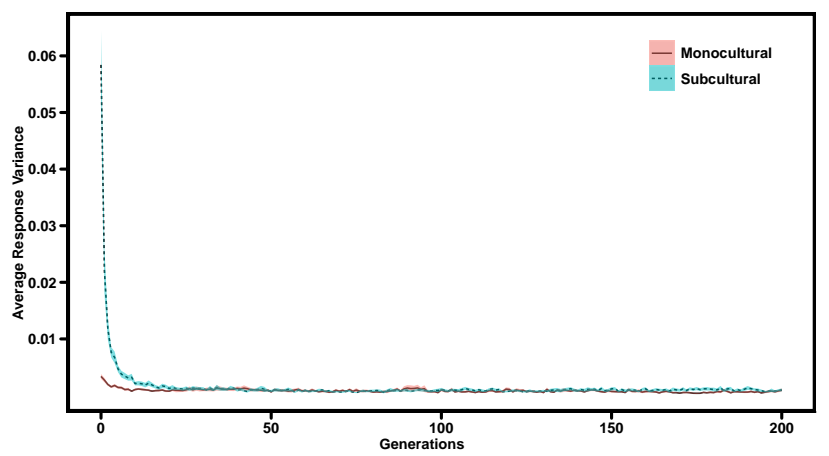

Figure 6: Behavioral diversity of the population's velocity actions for both monocultural and subcultural egalitarian social learning. Velocity converges quickly in both models, as there is rarely cause to move at any velocity below the maximum. This shows that subcultural ESL does not inhibit convergence when it is useful.

efficient and provides better results we incorporate it in our final algorithm.

To better understand why subcultural ESL is able to prevent premature convergence, the behavioral diversity of the population was analyzed after every generation. After each evaluation, a new instance of the simple world was generated and a set of input states was created corresponding to random locations, orientations, and velocities. For every agent, its output response (i.e., the desired change in velocity and orientation) was observed for each of the input states. Figures 6 and 7 show the average variance of the population response to each of the 100 input states after every generation in the complex domain, effectively measuring the behavioral diversity of the population. Velocity response converges for both models almost immediately, while orientation response remains more diverse in subcultural than in monocultural learning.

Although the orientation and velocity results may seem contradictory, they actually reflect a desirable property of subcultural learning. In the case of velocity, both the population and cultural algorithms quickly converge to low diversity. Since even at full speed the agents can sense plants up to ten timesteps away, there is no clear benefit to slowing down in the current domain and one would expect a strong strategy to always output the maximum value for velocity. Thus, preserving diversity in this area would be detrimental to the performance of the population. Conversely, the diversity of orientation should be preserved, as there is a tradeoff between turning toward an immediate but small reward, such as a single plant within one step, and turning toward a delayed reward, such as a cluster of plants a few steps away. This complexity suggests that the fitness landscape for orientation is likely filled with numerous local optima and diversity preservation should be beneficial to evolution.

The results of the diversity analysis suggest that the subcultural algorithm preserves diversity only when it is useful (e.g., orientation response), while not preventing convergence when diversity is unnecessary (e.g., velocity response).

The next experiment compares the ESL algorithm to a

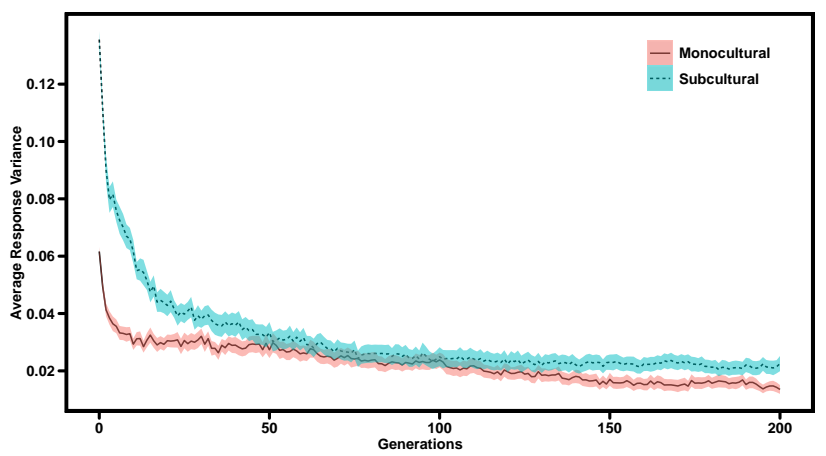

Figure 7: Behavioral diversity of the population's orientation actions for both monocultural and subcultural egalitarian social learning. There are many possible good responses for change in orientationtradeoffs between small, close rewards and large, distant rewards mean that exploring large portions of the search space can be fruitful. Subcultural ESL maintains a higher level of diversity and avoids converging to local optima.

benchmark student-teacher model and baseline neuroevolution in NEAT.

\subsection{ESL vs. Student-Teacher}

In the final set of experiments, subcultural ESL is compared to an on-line student-teacher learning algorithm inspired by the NEW TIES system [12]. The system utilizes a steady-state evolution in which at every timestep each agent probabilistically teaches the lowest-fitness member of the population within some radius, effectively forming geographical subcultures. The original system relies on a steady-state evolution where weaker agents eventually die out and do not have as much of a chance to propagate poor training examples as long-lasting agents. To adapt this approach to a generational EA, and to maximize the fairness of the comparison, a population of 10 subcultures is created with 10 agents each. At each time step, the current champion from each subculture teaches its group's lowest-fitness member on its most recent action regardless of whether the teaching agent received any reward.

It is worth noting that many social learning algorithms focus on off-line training, where teachers are the previous generation's champion(s). In these models, students are trained before the actual evaluation to mimic the champion(s), often with Gaussian noise added to the teacher's outputs to promote diversity in the population [9]. While such approaches may have also performed well in the foraging domain, they are not on-line learning algorithms. Rather, agents in these algorithms are static at evaluation time and do not learn from their own experiences or those of others. Thus, we chose to adapt the NEW TIES model to a generational EA as it is the most closely related approach in the literature.

Figures 8 and 9 show the results of the subcultural ESL algorithm compared to the student-teacher variant of NEW TIES and baseline neuroevolution. In the simple domain, all three approaches converge to solutions of approximately equivalent fitness after 200 generations. The student-teacher method converges to a near-optimal strategy slightly faster 


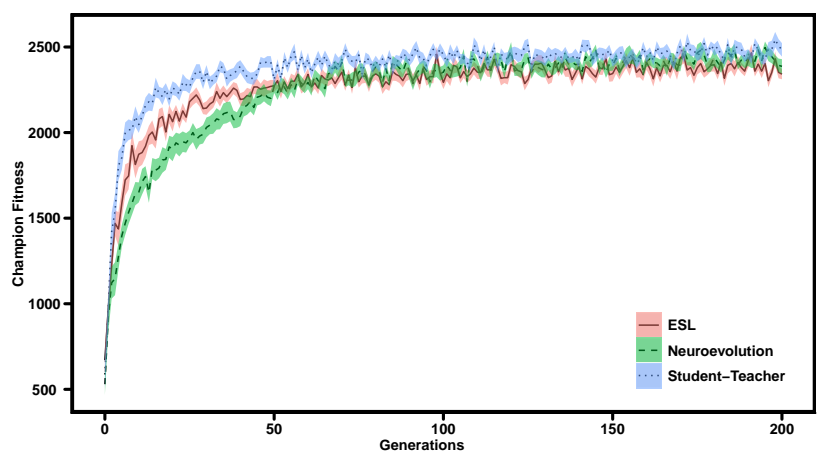

Figure 8: ESL compared to baseline neuroevolution, and student-teacher learning in the simple world. All strategies converge to solutions of similar quality, with the student-teacher approach converging slightly faster than ESL.

than ESL, though both are improvements over baseline neuroevolution. However, subcultural ESL converges to a nearoptimal solution much faster than the student-teacher variant in the complex world. ${ }^{2}$ The egalitarian approach is more than an order of magnitude faster in this domain, reaching a higher fitness by generation 50 than either the studentteacher or baseline neuroevolution methods achieve by generation 500 .

The contrasting performance in the two foraging domains highlights a key property of ESL - namely, its ability to combine a variety of sub-strategies into a single composite strategy. In the simple domain, with only a single plant type and relatively low dimensionality, such composite strategies are unnecessary as agents need only learn to navigate the world efficiently. Student-teacher methods are ideally suited for this class of tasks as they teach only a single strategy. In the complex world, ESL excels as it enables agents to combine effective sub-strategies for gathering or avoiding each plant type. Thus, ESL is likely best suited for domains where sophisticated agent behaviors are required.

The next section presents a discussion of the experimental results and indicates possible future directions for ESL.

\section{DISCUSSION AND FUTURE WORK}

The results of the above experiments in egalitarian social learning suggest that learning from strong actions, rather than high-fitness agents, is a powerful approach to social learning. The success of our algorithm in a given domain is predicated on two main user-defined parameters: the grouping of agents into subcultures and the criteria of the acceptability function.

For the experiments where subcultures were used, a simple division of 10 groups of equal size was chosen for the subculture creation strategy. Preliminary experiments showed that ESL results in the foraging domain were robust to moderate variations in the number of equal-sized subcultures, with 5 groups and 20 groups both demonstrating strong speedup compared to our benchmarks. However, it is possible that

\footnotetext{
${ }^{2}$ Note that in the foraging domain, near-optimal is often the best that can be accomplished as poisonous plants may be randomly laid out too close to nutritious ones, requiring an agent to eat either both or none.
}

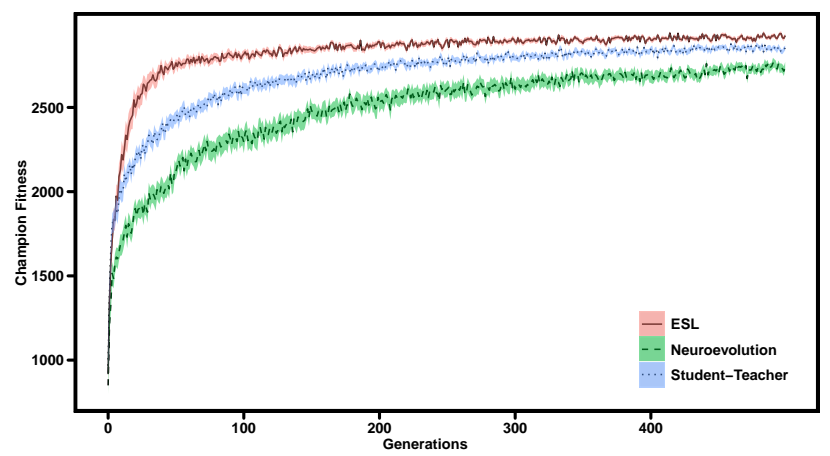

Figure 9: ESL compared to simple neuroevolution, and student-teacher learning in the complex world. ESL is more than an order of magnitude faster, reaching a higher fitness by generation 50 than either comparison method achieves by generation 500 .

equal-sized groups are not optimal or that a dynamic subculture creation algorithm that maximizes behavioral diversity would perform better; such issues will be addressed in future work.

Creating an acceptability function is a closely related task to that of creating a fitness function. Consequently, the performance of ESL depends on the ability of the user to craft a useful acceptability function. This task may require paying attention to the subtle details of one's domain as the definition of this function may not always be obvious. The acceptability function was intentionally kept simple in both foraging domains so as to demonstrate the strength of the general egalitarian learning approach. However, it is possible that employing a more sophisticated heuristic could speed up learning even more.

The robot foraging domain is well-suited for exploring extensions and improvements to egalitarian social learning. Whereas the current version only utilizes a single example at every time step, teaching more complex behaviors using reward-based approaches may require larger memory sizes. One potential future work direction is to introduce obstacles or walls that block the agent from immediately receiving the reward, necessitating that agents learn compound trajectories to navigate to the food.

Finally, we plan to expand our benchmark suite to include more student-teacher variants in the future. Student-teacher methods can be implemented in many ways, including offline learning [2], probabilistic teaching [12], and noisy examples [9], to name only a few. A more complete set of benchmarks will help us better understand the relative performance of each approach and how they compare to ESL both in the foraging domain and on future tasks.

\section{CONCLUSIONS}

This paper presented an egalitarian approach to social learning in evolutionary algorithms that enables agents to learn from high-quality actions. Unlike traditional social learning algorithms that follow a student-teacher model, ESL teaches agents based on acceptable actions taken by any agent in its subculture. By constraining teaching samples to those from the same subcultural group, ESL promotes diversity in the overall population and prevents premature 
convergence. Experiments in a complex robot foraging domain demonstrated that this approach is highly effective at quickly learning a near-optimal policy with Lamarckian evolution. Our results suggest that Egalitarian Social Learning is a strong technique and represents a promising new paradigm for social learning algorithms.

\section{REFERENCES}

[1] Mirror neurons and the simulation theory of mind-reading. Trends in Cognitive Sciences, 2(12):493 - 501, 1998.

[2] A. Acerbi and S. Nolfi. Social learning and cultural evolution in embodied and situated agents. In Artificial Life, 200\%. ALIFE'0\%. IEEE Symposium on, pages 333-340. IEEE, 2007.

[3] A. Acerbi and D. Parisi. Cultural transmission between and within generations. Journal of Artificial Societies and Social Simulation, 9(1), 2006.

[4] R. Arneson. Egalitarianism. 2009.

[5] C. Boehm. Hierarchy in the forest: The evolution of egalitarian behavior. Harvard Univ Pr, 2001.

[6] R. Byrne and A. Whiten. Machiavellian intelligence: Social expertise and the evolution of intellect in monkeys, apes, and humans. 1989.

[7] R. Dawkins. The selfish gene. Oxford University Press, 1976.

[8] M. de Oca, T. Stutzle, K. Van den Enden, and M. Dorigo. Incremental social learning in particle swarms. Systems, Man, and Cybernetics, Part B: Cybernetics, IEEE Transactions on, 41(2):368-384, 2011.

[9] D. Denaro and D. Parisi. Cultural evolution in a population of neural networks. M. marinaro and $r$. tagliaferri (eds), neural nets wirn-96. new york: Springer, pages 100-111, 1996.

[10] L. Erda, A. Whiten, P. Mellars, and K. Gibson. Egalitarianism and machiavellian intelligence in human evolution. 1996.

[11] L. Fogel, A. Owens, and M. Walsh. Artificial intelligence through simulated evolution. 1966.

[12] E. Haasdijk, P. Vogt, and A. Eiben. Social learning in population-based adaptive systems. In Evolutionary Computation, 2008. CEC 2008. (IEEE World Congress on Computational Intelligence). IEEE Congress on, pages 1386-1392. IEEE, 2008.

[13] E. Herrmann, J. Call, M. Hernández-Lloreda, B. Hare, and M. Tomasello. Humans have evolved specialized skills of social cognition: The cultural intelligence hypothesis. science, 317(5843):1360, 2007.

[14] K. Holekamp. Questioning the social intelligence hypothesis. Trends in cognitive sciences, 11(2):65-69, 2007.

[15] N. Humphrey. The social function of intellect. Growing points in ethology, pages 303-317, 1976.

[16] J. Kennedy and R. Eberhart. Particle swarm optimization. In Neural Networks, 1995. Proceedings., IEEE International Conference on, volume 4, pages 1942-1948. IEEE, 1995.

[17] K. Ku, M. Mak, and W. Siu. A study of the lamarckian evolution of recurrent neural networks. Evolutionary Computation, IEEE Transactions on, 4(1):31-42, 2000.
[18] P. Moscato. On evolution, search, optimization, genetic algorithms and martial arts: Towards memetic algorithms. Caltech Concurrent Computation Program, C3P Report, 826:1989, 1989.

[19] G. Onwubolu and B. Babu. New optimization techniques in engineering, volume 141. Springer Verlag, 2004.

[20] D. Parisi. Cultural evolution in neural networks. IEEE Expert, 12(4):9-14, 1997.

[21] R. Reynolds. An introduction to cultural algorithms. In Proceedings of the Third Annual Conference on Evolutionary Programming, pages 131-139. World Scientific, 1994.

[22] D. Rumelhart, G. Hinton, and R. Williams. Learning representations by back-propagating errors. Nature, 323(6088):533-536, 1986.

[23] G. Simpson. The baldwin effect. Evolution, $7(2): 110-117,1953$.

[24] K. Stanley and R. Miikkulainen. Evolving neural networks through augmenting topologies. Evolutionary computation, 10(2):99-127, 2002.

[25] M. Tomasello. The cultural origins of human cognition. Harvard Univ Pr, 1999.

[26] P. Vogt and E. Haasdijk. Modeling social learning of language and skills. Artificial Life, 16(4):289-309, 2010.

[27] C. Watkins and P. Dayan. Q-learning. Machine learning, 8(3):279-292, 1992.

[28] P. Werbos. Backpropagation through time: what it does and how to do it. Proceedings of the IEEE, 78(10):1550-1560, 1990.

[29] S. Whiteson and P. Stone. Evolutionary function approximation for reinforcement learning. The Journal of Machine Learning Research, 7:877-917, 2006. 